\title{
Consumer Wearables and Affective Computing for Wellbeing Support
}

\author{
Stanisław Saganowski \\ stanislaw.saganowski@pwr.edu.pl \\ Faculty of Computer Science and \\ Management, Wroclaw University of \\ Science and Technology \\ Wroclaw, Poland \\ Patrycja Jakimów \\ Faculty of Computer Science and \\ Management, Wroclaw University of \\ Science and Technology \\ Wroclaw, Poland \\ Anna Dutkowiak \\ Faculty of Electronics, Wroclaw \\ University of Science and Technology \\ Wroclaw, Poland
}

\author{
Przemysław Kazienko \\ Faculty of Computer Science and \\ Management, Wroclaw University of \\ Science and Technology \\ Wroclaw, Poland
}

\author{
Joanna Komoszyńska \\ Faculty of Computer Science and \\ Management, Wroclaw University of \\ Science and Technology \\ Wroclaw, Poland \\ Adam Polak \\ Faculty of Electronics, Wroclaw \\ University of Science and Technology \\ Wroclaw, Poland
Michał Ujma
Capgemini
Wroclaw, Poland

\author{
Maciej Dzieżyc \\ Faculty of Computer Science and \\ Management, Wroclaw University of \\ Science and Technology \\ Wroclaw, Poland
}

\author{
Weronika Michalska \\ Faculty of Computer Science and \\ Management, Wroclaw University of \\ Science and Technology \\ Wroclaw, Poland \\ Adam Dziadek
Capgemini
Wroclaw, Poland
}

\begin{abstract}
Wearables equipped with pervasive sensors enable us to monitor physiological and behavioral signals in our everyday life. We propose the WellAff system able to recognize affective states for wellbeing support. It also includes health care scenarios, in particular patients with chronic kidney disease suffering from bipolar disorders. For the need of a large-scale field study, we revised over 50 off-the-shelf devices in terms of usefulness for emotion, stress, meditation, sleep, and physical activity recognition and analysis. Their usability directly comes from the types of sensors they possess as well as the quality and availability of raw signals. We found there is no versatile device suitable for all purposes. Using Empatica E4 and Samsung Galaxy Watch, we have recorded physiological signals from 11 participants over many weeks. The gathered data enabled us to train a classifier that accurately recognizes strong affective states.
\end{abstract}

\section{KEYWORDS}

wellbeing, wearables, affective computing, wellaff system, affect recognition, sleep, physical activity, meditation, stress, smartwatch, wristband, armband, fitband

Permission to make digital or hard copies of all or part of this work for personal or classroom use is granted without fee provided that copies are not made or distributed for profit or commercial advantage and that copies bear this notice and the full citation on the first page. Copyrights for components of this work owned by others than ACM must be honored. Abstracting with credit is permitted. To copy otherwise, or republish, to post on servers or to redistribute to lists, requires prior specific permission and/or a fee. Request permissions from permissions@acm.org.

MobiQuitous '20, December 7-9, 2020, Darmstadt, Germany

(c) 2018 Association for Computing Machinery.

ACM ISBN 978-1-4503-8840-5/20/11.

https://doi.org/10.1145/3448891.3450332
ACM Reference Format:

Stanisław Saganowski, Przemysław Kazienko, Maciej Dzieżyc, Patrycja Jakimów, Joanna Komoszyńska, Weronika Michalska, Anna Dutkowiak, Adam Polak, Adam Dziadek, and Michał Ujma. 2020. Consumer Wearables and Affective Computing for Wellbeing Support. In MobiQuitous 2020 - 17th EAI International Conference on Mobile and Ubiquitous Systems: Computing, Networking and Services (MobiQuitous '20), December 79, 2020, Darmstadt, Germany. ACM, New York, NY, USA, 6 pages. https: //doi.org/10.1145/3448891.3450332

\section{INTRODUCTION}

Each iteration of modern smartwatches, wristbands, armbands, fitbands, headbands, chest straps, and patches is more powerful, precise, comfortable, and useful. At the same time, wearables are more affordable and easily available, ultimately becoming pervasive. Therefore, they may be applied in more and more domains. Their portability and low costs enable us to perform not only research in the lab but also large-scale field studies.

Consequently, consumer wearables connected with smartphones are a perfect instrument for various wellbeing-related solutions. In particular, they may monitor our affective states and accordingly support decision making like environment adaptation (calming lighting if you are over-excited), alerting parents when a distant child is experiencing difficult times, warning very overweight people not to eat when they are having strong emotions or reporting to nephrologists about frequent changes in mood in patients with chronic kidney disease (CKD) or after kidney transplantation in order to adjust treatment or drug dosing.

To enable the aforementioned applications, we propose the WellAff system, Section 4. Its primary purpose is to monitor and 
Table 1: The usefulness of consumer wearables in emotion (Emo), stress (Str), meditation (Md), sleep (Slp), and physical activity (Act) analysis. (Phy. raw sign.) denotes the availability of raw physiological signals; $\left({ }^{*}\right)$ marks wearables tested by us; $\left({ }^{* *}\right)$ denotes the ECG sensor unlocked only in the South Korean market so far. Factors considered in grading: richness, sampling, and availability of relevant data, domain-related convenience, battery life.

\begin{tabular}{|c|c|c|c|c|c|c|c|c|c|c|}
\hline Device* $^{*}$ & Type & Release & Sensors & Phy. raw sign. & Other data & Emo & $\overline{\text { Str }}$ & Md & Slp & $\overline{\text { Act }}$ \\
\hline $\begin{array}{l}\text { Samsung Galaxy } \\
\text { Watch } 3^{*}\end{array}$ & $\begin{array}{l}\text { Smart- } \\
\text { watch }\end{array}$ & 2020.08 & $\begin{array}{c}\text { PPG, ECG }{ }^{* *} \text { ACC, GYRO, } \\
\text { BAR, AL, MIC, GPS }\end{array}$ & BVP & $\begin{array}{l}\text { HR, ACC, GYRO, BAR, AL, } \\
\text { MIC, GPS, STP }\end{array}$ & ++ & ++ & + & ++ & +++ \\
\hline Apple Watch $5^{*}$ & $\begin{array}{l}\text { Smart- } \\
\text { watch }\end{array}$ & 2019.09 & $\begin{array}{c}\text { PPG, ECG, ACC, GYRO, } \\
\text { BAR, MIC, GPS }\end{array}$ & - & $\begin{array}{l}\text { HR, ACC, GYRO, BAR, } \\
\text { MIC, GPS, STP, CAL }\end{array}$ & + & ++ & + & ++ & +++ \\
\hline Fossil Gen $5^{*}$ & $\begin{array}{l}\text { Smart- } \\
\text { watch }\end{array}$ & 2019.08 & $\begin{array}{l}\text { PPG, ACC, GYRO, ALT, } \\
\text { AL, MIC, GPS }\end{array}$ & BVP & $\begin{array}{c}\text { HR, ACC, GYRO, ALT, AL, } \\
\text { MIC, GPS, STP }\end{array}$ & ++ & ++ & + & ++ & +++ \\
\hline Garmin Fenix 6X Pro & $\begin{array}{l}\text { Smart- } \\
\text { watch }\end{array}$ & 2019.08 & $\begin{array}{c}\text { PPG, SpO2, ACC, GYRO, } \\
\text { ALT, AL, GPS }\end{array}$ & BVP, SpO2 & $\begin{array}{c}\text { HR, ACC, GYRO, ALT, AL, } \\
\text { GPS, STP }\end{array}$ & ++ & & + & ++ & +++ \\
\hline $\begin{array}{l}\text { Samsung Galaxy } \\
\text { Watch }^{*}\end{array}$ & $\begin{array}{l}\text { Smart- } \\
\text { watch }\end{array}$ & 2019.08 & $\begin{array}{c}\text { PPG, ACC, GYRO, BAR, } \\
\text { AL, MIC, GPS }\end{array}$ & BVP & $\begin{array}{c}\text { HR, ACC, GYRO, BAR, AL, } \\
\text { MIC, GPS, STP }\end{array}$ & ++ & ++ & + & ++ & +++ \\
\hline Polar OH1 & Armband & 2019.03 & PPG, $\mathrm{ACC}$ & BVP & PPI, ACC & ++ & ++ & + & ++ & ++ \\
\hline Samsung Galaxy Fit E* & Fitband & 2019.02 & PPG, ACC & - & HR & - & + & + & + & + \\
\hline Garmin HRM-DUAL & Chest strap & 2019.01 & ECG & ECG & RRI & ++ & ++ & + & + & + \\
\hline Muse $2^{*}$ & $\begin{array}{c}\text { EEG } \\
\text { headband }\end{array}$ & 2019.01 & $\begin{array}{c}\text { EEG, PPG, SpO2, ACC, } \\
\text { GYRO }\end{array}$ & EEG, BVP, SpO2 & HR, ACC, GYRO & ++ & ++ & +++ & + & - \\
\hline Fitbit Charge $3^{*}$ & Fitband & 2018.10 & PPG, ACC, GYRO, ALT & - & HR, ACC, ALT & + & ++ & + & ++ & ++ \\
\hline $\begin{array}{c}\text { Garmin VivoActive } 3 \\
\text { Music* }^{*}\end{array}$ & $\begin{array}{l}\text { Smart- } \\
\text { watch }\end{array}$ & 2018.06 & $\begin{array}{c}\text { PPG, ACC, GYRO, BAR, } \\
\text { GPS }\end{array}$ & - & $\begin{array}{c}\text { HR, PPI, RSP, ACC, STP, } \\
\text { CAL }\end{array}$ & + & ++ & + & ++ & ++ \\
\hline Oura ring ${ }^{*}$ & Smart ring & 2018.04 & PPG, ACC, GYRO, TERM & - & HR, PPI, SKT, SP & - & ++ & + & +++ & + \\
\hline Moodmetric $^{*}$ & Smart ring & 2017.12 & EDA, ACC & EDA & STP & + & ++ & - & + & - \\
\hline DREEM & $\begin{array}{c}\text { EEG } \\
\text { headband }\end{array}$ & 2017.06 & EEG, PPG, SpO2, ACC & EEG, BVP, SpO2 & $\mathrm{HR}, \mathrm{ACC}$ & ++ & & +++ & ++ & - \\
\hline Polar H10 & Chest strap & 2017.03 & ECG, ACC & ECG & RRI, ACC & ++ & ++ & + & ++ & ++ \\
\hline VitalPatch & Chest patch & 2016.03 & ECG, ACC, TERM & ECG, SKT & HR, RRI, EDR, STP & ++ & ++ & + & ++ & + \\
\hline Sony SmartBand 2 & Fitband & 2015.09 & PPG, ACC & BVP & HR, PPI, ACC & + & ++ & + & ++ & ++ \\
\hline Empatica E4* & Wristband & 2015 & PPG, EDA, ACC, TERM & BVP, EDA, SKT & HR, PPI, ACC, tags & +++ & +++ & + & +++ & ++ \\
\hline Microsoft Band 2 & Smartband & 2014.10 & $\begin{array}{l}\text { PPG, EDA, ACC, GYRO, } \\
\text { TERM, BAR, ALT, AL, UV }\end{array}$ & BVP, EDA, SKT & $\begin{array}{l}\text { HR, PPI, ACC, GYRO, BAR, } \\
\text { ALT, AL, STP, CAL, UV }\end{array}$ & +++ & +++ & + & +++ & ++ \\
\hline Samsung Gear Live & Fitband & 2014.06 & PPG, ACC, GYRO & BVP & HR, ACC, GYRO, STP & ++ & ++ & + & ++ & ++ \\
\hline Philips DTI-2 & Wristband & 2014.03 & $\begin{array}{c}\text { EDA, ACC, TERM, AL, } \\
\text { AT }\end{array}$ & EDA & ACC, TEMP, AL, AT & + & ++ & - & ++ & + \\
\hline
\end{tabular}

recognize affective states like emotions as well as their changes in everyday life based on physiological sensor signals provided by off-the-shelf devices.

For that purpose, we revised consumer wearables. The existing literature has focused on usefulness of commercially available devices in one [7] or two domains [36], or on validity of wearable sensors [13]. In this work, however, we evaluate over 50 off-theshelf wearable devices in terms of their usefulness and applicability in emotion and stress recognition, as well as sleep, meditation, and activity monitoring, Section 3 . We focus on the sensors built into the device, the signals they provide, and the availability of the raw data recorded or extracted with these sensors. This survey enabled us to select the most suitable devices for our further studies within the WellAff system: auxiliary Samsung Galaxy Watch and Empatica E4, as well as the target - Samsung Galaxy Watch 3.

An essential component of every machine learning-based recognition system is annotated data. There are only a few studies about emotion recognition in the field [38]. Therefore, we decided to perform a large-scale real life study. It was split into three phases, Fig. 1: (1) data accumulation and a reasoning system about strong affective states, (2) recognition of these strong states and their more detailed self-annotations, and (3) final application of complex states recognition and change detection for wellbeing support.

One of the main challenges to overcome was: how to gather data about affect experienced in real life without a significant impact of the questioning procedure on experiencing itself? Hence, we believe that the annotation of affective states in real life should be as little intrusive as possible. Therefore, we apply in the WellAff system the concept of Ecological Momentary Assessment (EMA) [43], which is in line with Descriptive Experience Sampling (DES). DES captures the inner experience of subjects in their natural environments using a random beeper invoking rare subject surveying [24]. We implemented this by means of randomness and decreased questioning frequency. EMA, in turn, is a data collection method in real-time and in real-world settings to avoid retrospective biases and gather ecologically valid data. EMA is particularly suited for studying substance use - in our case, strong affect events.

\section{SIGNALS IN EMOTION, MEDITATION, STRESS, SLEEP, AND ACTIVITY ANALYSIS}

Wearables are equipped with sensors that provide physiological, behavioral, and environmental data, Tab. 1. These sensors are EEG 
- electroencephalogram, PPG - photoplethysmograph ${ }^{1}$ delivering BVP - Blood Volume Pulse signal and derived signal PPI - peak-topeak intervals (a.k.a. HRV - heart rate variability, or IBI - interbeat interval), ECG - electrocardiograph providing RRI - R-R intervals, EDA - electrodermal activity sensor (a.k.a GSR - galvanic skin response), $\mathrm{SpO} 2$ - blood oxygen saturation sensor, ACC - accelerometer, GYRO - gyroscope, TERM - thermometer providing SKT - skin temperature, BAR - barometer, ALT - altimeter, AL - ambient light sensor, AT - ambient temperature sensor, MIC - microphone, MAG - magnetometer, UV - ultraviolet, and GPS. They can also provide other data derived from the monitored signals: HR - heart rate (extracted either from BVP/PPI or ECG/RRI), STP - number of steps, RSP - respiration rate, EDR - RSP from ECG, CAL - calories burned, SP - sleep phases.

Emotions. The EEG outperforms other signals in terms of usefulness for emotion recognition [31, 45]. However, recently, studies tend to employ wearables [38], which provide various bio-signals and additional environmental data. Most often, ECG/BVP and EDA signals are utilized [16, 32, 41]. Those signals can be supplemented with ACC, GYRO SKT, RSP [2, 23, 27, 39, 40], as well as with UV, GPS, and MIC data [25].

Stress. Bio-signals related to stress include EEG, ECG, BVP, EDA, SKT, and RSP $[9,18]$. The best stress detection accuracy in the field can be achieved when using ECG/BVP and EDA signals together $[10,17,19]$. Albeit, using the ECG/BVP or EDA signal solely also provides satisfactory results $[48,49]$.

Meditation. The most appropriate physiological signal to study meditation is EEG [21, 22, 42]. Other useful signals are ECG [1, 26], BVP [47], and EDA [35].

Sleep. There are two main methods for gathering data in sleep studies: polysomnography (PSG) and actigraphy (ACG) [20]. A gold standard is PSG, which uses advanced medical equipment to measure EEG, EOG, EMG, ECG. However, due to the complexity, size, wiring, and cost of the equipment, PSG cannot be applied outside the lab. For field studies, ACG is an appropriate measurement method based on the movement signals, i.e., ACC data. More recent studies also utilize BVP $[6,14]$, SKT [46], and MIC [8, 11]. Data from SpO2, ECG, RSP, and MIC allow us to diagnose the sleep apnea $[15,28,33]$.

Physical activity research commonly focuses on the following problems: identification, tracking, and quantification. The most frequently used sensors, providing data to tackle these problems are ACC, GYRO, and GPS [3, 4, 30, 44], as well as PPG [5, 29, 37], and ECG [34].

\section{CONSUMER WEARABLES FOR ACTIVITY AND AFFECTIVE COMPUTING}

When considering whether a device is appropriate for lab or field studies related to a given problem, many factors have to be taken into account. The most important is the ability to provide relevant and rich data in a raw format. Many producers develop dedicated applications for end-users to allow them convenient monitoring of the data collected by various sensors. However, this data is usually processed in some way (e.g., aggregated, filtered, smoothed)

\footnotetext{
${ }^{1}$ Some producers like Samsung in their Galaxy Watch series provide the raw PPG signal in the form of light intensity stream.
}

and very rarely available to download. Thus, not useful for the research purpose. Some manufacturers provide the ability to access raw data by integrating with a device over Bluetooth or another protocol, e.g., Empatica E4, Garmin HRM-DUAL, Moodmetric. Such integration is sufficient to gather data for the research but still has some limitations, e.g., the sensors' sampling frequency cannot be adjusted. Only a few producers provide an SDK (software development kit) that allows us to develop our own software and embed it into devices, e.g., Samsung's smartwatches running Tizen operating system (OS), and Wear OS devices. See Tab. 1, column Phy. raw sign. for the list of raw physiological signals provided by particular consumer devices. Overall, the more signals/sensors related to a considered problem, the better.

Tab. 1 only contains devices that are portable and grant access to the gathered data. Of course, the portability argument is subjective. Ten years ago, a backpack with sensors was considered portable. Today, portables have to be small and handy. Perhaps, in five years, portable would mean barely visible or even implanted.

Raw signals, especially sampled with high frequency, provide more flexibility in research; hence, their availability is essential. Portability, in turn, is crucial for field studies in particular longterm ones. Some devices provide signals in real-time, whereas the others after the session termination. All wearables in Tab. 1, except DTI-2, are equipped with Bluetooth Low Energy (BLE); few also provide Wi-Fi, LTE, and NFC connectivity. We examined devices marked with * in terms of raw signals availability. For other devices, we relied on the official producers' or other external information.

To sum up, there is no off-the-shelf device equipped well enough for a proper analysis in all domains, i.e., emotion, stress, meditation, sleep, and physical activity. Producers design them for specific market needs and use sensors and electronic units of different quality and often provide access only to selected data. Moreover, the available signals may have too low sampling frequency. For example, the averaged over 5 mins PPI and HR in Oura Ring is insufficient for accurate emotion recognition (-) but is acceptable for approximate stress detection $(++)$. Furthermore, with additional sleep-related data Oura is very good for sleep analysis $(+++)$. The same refers to Apple Watch 5 that provides HR data every 5 secs (and no other physiological signals) - too rarely for emotions but good enough for activity studies $(+++)$.

The best wearables for emotion, stress, and sleep research appear to be the relatively old Empatica E4 and Microsoft Band 2. The former will be replaced with EmbracePlus this year; the latter is no longer supported. EEG headbands, Muse 2, and DREEM are the best choice for meditation. Although DREEM is intended for sleep studies, we argue that it is not very comfortable. Oura Ring is more suitable for undisturbed sleep analysis. The smartwatches by Apple, Fossil, Garmin, and Samsung have proper sensors and provide the best data for activity investigations.

We have also considered some other devices, like Emotiv Epoc $+{ }^{*}$, NeuroSky, emWave2, Honor Band $4^{*}$, Xiaomi Mi Band $3^{*}$, Xiaomi Mi Band 5*, Polar A370*, Fitbit Blaze*, and 20 others. However, they do not offer access to the data or have other drawbacks. Therefore, they have not been included in Tab. 1 .

In our studies focused on the affect recognition, we have decided to use a few Empatica E4 devices and dozens of Samsung Galaxy Watches. Empatica E4 offers the greatest number of sensors among 
the devices available in the market but is also a few times more expensive than smartwatches. On the other hand, Samsung Galaxy Watch lacks only the EDA and SKT sensors, but offers some other data like BAR or AL (eventually, should also offer ECG), and allows us to build custom watch applications.

\section{WELLAFF: A SYSTEM FOR WELLBEING SUPPORT BASED ON AFFECTIVE COMPUTING}

The general idea of the WellAff system, including its development phases, is depicted in Fig. 1. The goal of the first phase is to train a classification model able to recognize strong affective states experienced in everyday life based on physiological signals from wearables (smartwatches, wristbands). We have developed a watch application for Samsung Galaxy watches that allows the participants to mark both strong affective states and neutral ones (low arousal). An analogous, built-in functionality is provided by Empatica E4 by means of the dedicated button. Having physiological signals annotated with very intensive and neutral states, we can build a binary classifier recognizing whether a participant is currently experiencing a strong affect or not.

The trained classifier is directly used as a predictor in the second phase. It is a crucial part of the integrated smartwatch and smartphone application. It continuously monitors physiological and behavioral signals. If, based on them, it recognizes a strong affect, a notification appears on the watch with a request to confirm the prediction. The participants may either reject such a suggestion (then we mark a false positive prediction) or accept it. If they agree, the phone application provides the EMA-type questionnaire [43] - a list of affective states along with the open text option. Additionally, to make the system less intrusive and avoid too frequent surveying, we apply some randomness and minimal idle time between consecutive questioning. In this way, we are able to identify moments of experiencing strong affects in real life without much interfering. As a result, we obtain a new data set consisting of signals and new, more comprehensive manual annotations. Some affective states like emotions can often co-occur. Having this in mind and based on the data collected, we build various prediction models with distinct outputs: binary, multi-class, multi-label, regression, multi-dimensional, structured output, e.g., a sequence of states/values. The model selection depends on the use-case (wellbeing support) that we want to apply in the final phase of the WellAff system.

The learned models are used in the third phase as a component of another wearable and mobile application. Similarly to the previous stage, the system is able to analyze signals provided by wearable sensors and to provide more complex predictions: different co-occurring affective states, their levels, and transitions meaningful changes. These real-time predictions are used in various applications related to wellbeing, personal, and health support. For example, bipolar disorders and related emotional distress may result from brain metabolic disturbances caused by uremic toxins accumulation. This, in turn, can be a symptom of Chronic Kidney Disease (CKD) or problems with treatment after renal transplantation. Being able to recognize the mixed affective states at an early stage, we could suggest CKD patients to go on dialysis, thus, avoiding significant toxins accumulation and consequently improving their mood and quality of life.

So far, we have completed the first phase with preliminary results, see Section 5.

\section{AFFECT RECOGNITION IN EVERYDAY LIFE - EXPERIMENTAL RESULTS}

To implement the entire WellAff idea, we started the first phase in spring 2020. For the purpose of all our WellAff-related studies, we received bioethics committee approval no. 149/2020 from Wroclaw Medical University.

\subsection{Learning Dataset Collection}

In the first phase, we have used Empatica E4 and Samsung Galaxy Watch 2019 wearables. The Empatica is among the most appropriate devices for affect recognition, but not very convenient for the enduser, while Samsung Galaxy Watch is user-friendly and lacks only EDA and SKT signals, see Tab. 1. The participants used a dedicated smartwatch application to mark with timestamps the affective states they experience in their life. Additionally, they filled up an online questionnaire to report the type of the state (especially strong affect vs. neutral) and a possible delay between the experiencing affect and marked timestamp. If the delay exceeds 60 seconds, the events are excluded from the study, because in our opinion, it is hard to accurately estimate the delay after a couple of minutes from the strong affective event.

At the moment, we have gathered 294 affective samples (206 strong affect, 75 - neutral or calm state, 13 samples were marked by mistake, therefore excluded) from six female and five male subjects, aged 23-53. During the three-month span, we have recorded over 3000 hours for each physiological and behavioral signal. Finally, we used signals from the following sensors: PPG (light intensity for Samsung Galaxy Watch, BVP for Empatica), ACC, TERM, and EDA (Empatica only), AL, GYRO, and BAR (Samsung only). An example of physiological signals during a strong affect - fear for the choked child is depicted in Fig. 2. The participant's body response in the form of a peak in the EDA signal can be easily observed. The data collection in this phase is still on-going, and new samples are gathered every day.

\subsection{Model for Strong Affect Recognition}

From all the recordings, the 60 -second signal windows preceding the reported affective states have been extracted. Then, the signals were pre-processed using winsorization, Butterworth low-pass filter with $10 \mathrm{~Hz}$ cut-off, and min-max normalization. The stratified sampling has been applied to divide 281 samples into training and test sets with 80:20 ratio and 5-fold cross-validation. The cases in the training set were balanced using the SMOTE [12] method. Next, over 60 features were extracted from BVP, EDA, SKT, and ACC signals using BioSPPy ${ }^{2}$, pyphysio ${ }^{3}$, and $\mathrm{SciPy}^{4}$ libraries. After feature selection and reduction, the binary classification (strong affect vs. neutral state) has been performed. The initial calculations

\footnotetext{
${ }^{2}$ https://biosppy.readthedocs.io

${ }^{3}$ https://github.com/MPBA/pyphysio

${ }^{4}$ https://www.scipy.org
} 


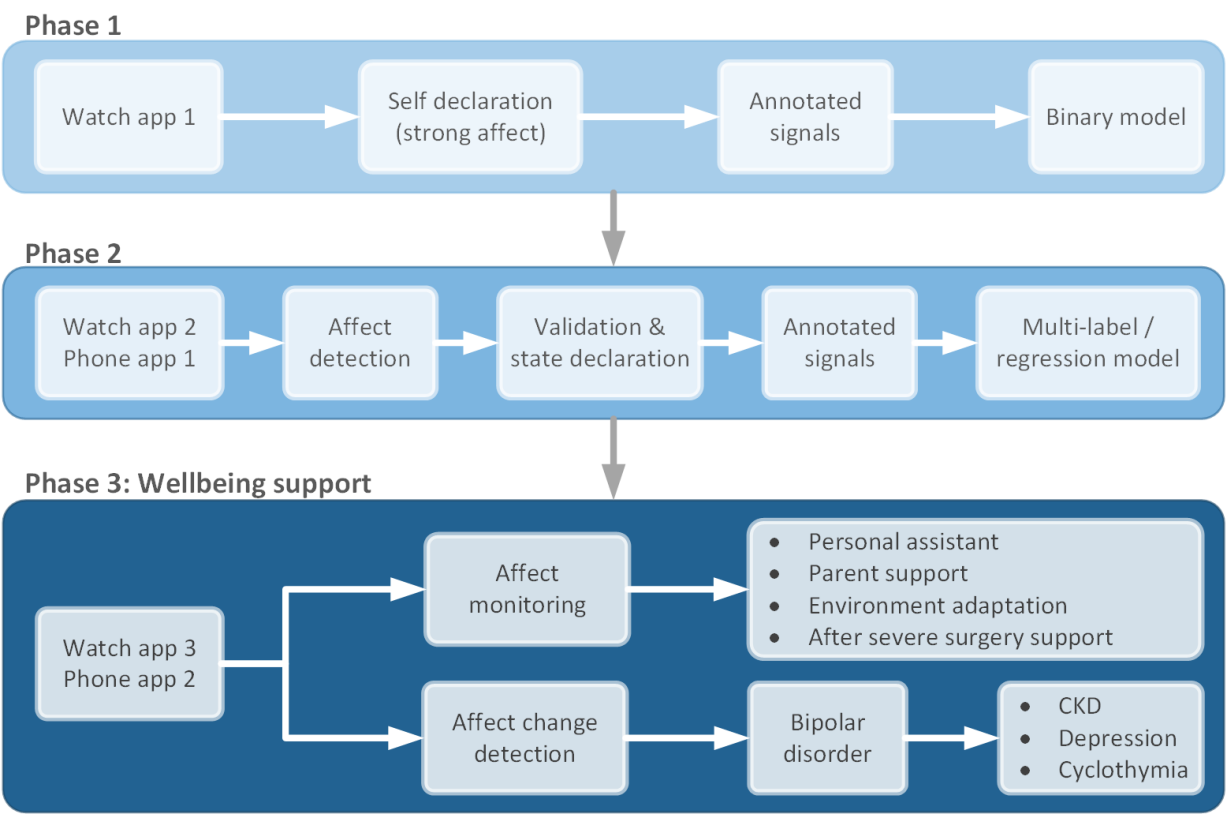

Figure 1: A WellAff system for wellbeing support through monitoring and recognition of affective states in everyday life.

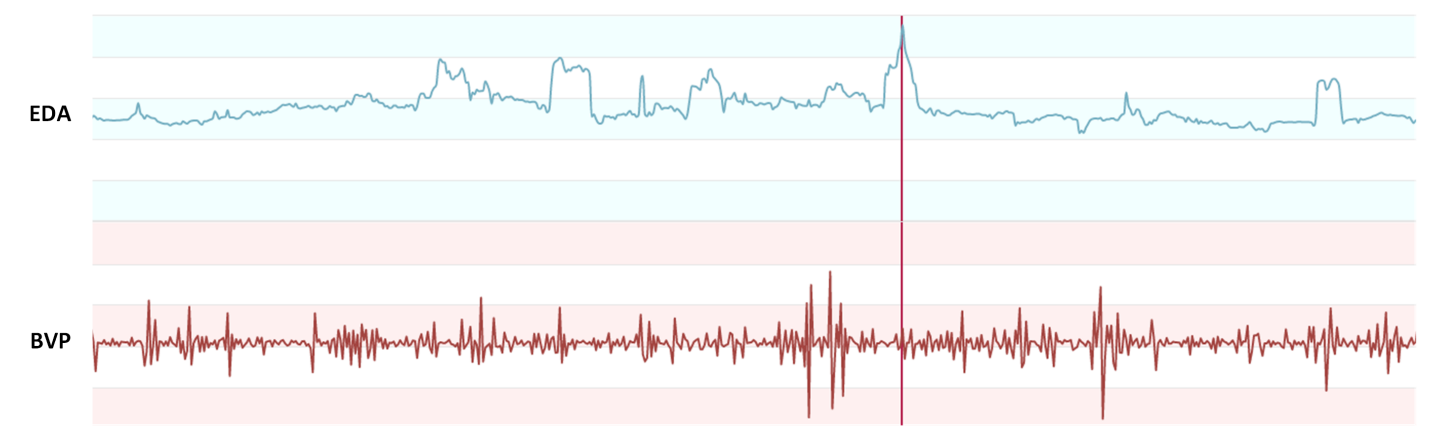

Figure 2: An example of strong affect (fear for the choked child) experienced in real life and marked by the participant. The body response in term of peak of the EDA signal can be easily observed.

performed with the tree-based models wrapped with the AdaBoost ensemble algorithm provided $91 \%$ F1-measure efficiency.

\section{CONCLUSIONS}

Consumer wearables are yet to match the medical-level devices in terms of sensor and signal quality. The main advantage of wearables is their portability, ubiquity, and multiple sensors enabling large-scale multimodal studies in everyday life. There are suitable wearables for each of the research topics considered in this paper. The initial results suggest that we can benefit from the use of wearables in wellbeing support.

The nearest future work will cover: (i) additional sampling of neutral states to deliver a better class balance, (ii) further collection of new samples with new subjects and diverse conditions, (iii) training and validation of the end-to-end multimodal deep learning models as well as their simplification for reasoning purposes on smartwatches and smartphones (some preliminary results have already been obtained), (iv) implementation of the second and third phase, (v) gathering data from CKD-suffering patients.

\section{ACKNOWLEDGMENTS}

This work was partially supported by the National Science Centre, Poland, project no. 2016/21/B/ST6/01463; and the statutory funds of the Department of Computational Intelligence, Wroclaw University of Science and Technology.

\section{REFERENCES}

[1] H Alawieh, Z Dawy, E Yaacoub, and et al. 2019. A Real-Time ECG Feature Extraction Algorithm for Detecting Meditation Levels within a General Measurement Setup. In 41st Annual Int. Conf. of the IEEE Eng. in Med. and Biol. Soc. IEEE, 99-103.

[2] Amani Albraikan, Basim Hafidh, and Abdulmotaleb El Saddik. 2018. iAware: A Real-Time Emotional Biofeedback System Based on Physiological Signals. IEEE Access 6 (2018), 78780-78789. 
[3] H Allahbakhshi, L Conrow, B Naimi, and R Weibel. 2020. Using Accelerometer and GPS Data for Real-Life Physical Activity Type Detection. Sensors 20, 3 (2020), 588.

[4] G Aroganam, N Manivannan, and D Harrison. 2019. Review on wearable technology sensors used in consumer sport applications. Sensors 19, 9 (2019), 1983.

[5] KR Arunkumar and M Bhaskar. 2020. Heart rate estimation from wrist-type photoplethysmography signals during physical exercise. Biomedical Signal Processing and Control 57 (2020), 101790.

[6] Mahmoud Assaf, Aïcha Rizzotti-Kaddouri, and Magdalena Punceva. 2018. Sleep detection using physiological signals from a wearable device. In EAI International Conference on IoT Technologies for HealthCare. Springer, 23-37.

[7] MI Cajita, CE Kline, and et al. 2020. Feasible but Not Yet Efficacious: a Scoping Review of Wearable Activity Monitors in Interventions Targeting Physical Activity, Sedentary Behavior, and Sleep. Current Epidem. Rep. 7, 1 (2020), 25-38.

[8] Burçin Camc1, Cem Ersoy, and Hakan Kaynak. 2019. Abnormal respiratory event detection in sleep: a prescreening system with smart wearables. Fournal of biomedical informatics 95 (2019), 103218

[9] Yekta Said Can, Bert Arnrich, and Cem Ersoy. 2019. Stress detection in daily life scenarios using smart phones and wearable sensors: A survey. Fournal of biomedical informatics (2019), 103139.

[10] Yekta Said Can, Niaz Chalabianloo, Deniz Ekiz, and Cem Ersoy. 2019. Continuous stress detection using wearable sensors in real life: Algorithmic programming contest case study. Sensors 19, 8 (2019), 1849.

[11] Rossana Castaldo, Marta Prati, Luis Montesinos, Vishwesh Kulkarni, Micheal Chappell, Helen Byrne, Pasquale Innominato, Stephen Hughes, and Leandro Pecchia. 2019. Investigating the Use of Wearables for Monitoring Circadian Rhythms: A Feasibility Study. In International Conference on Biomedical and Health Informatics. Springer, 275-280.

[12] Nitesh V Chawla, Kevin W Bowyer, Lawrence O Hall, and W Philip Kegelmeyer. 2002. SMOTE: synthetic minority over-sampling technique. fournal of artificial intelligence research 16 (2002), 321-357.

[13] Gloria Cosoli, Susanna Spinsante, and Lorenzo Scalise. 2020. Wrist-worn and chest-strap wearable devices: systematic review on accuracy and metrological characteristics. Measurement (2020), 107789

[14] Massimiliano de Zambotti, Leonardo Rosas, Ian M Colrain, and Fiona C Baker 2019. The sleep of the ring: comparison of the OURA sleep tracker against polysomnography. Behavioral sleep medicine 17, 2 (2019), 124-136.

[15] Urtnasan Erdenebayar, Yoon Ji Kim, Jong-Uk Park, Eun Yeon Joo, and KyoungJoung Lee. 2019. Deep learning approaches for automatic detection of sleep apnea events from an electrocardiogram. Computer methods and programs in biomedicine 180 (2019), 105001.

[16] Luz Fernández-Aguilar, Arturo Martínez-Rodrigo, José Moncho-Bogani, Antonio Fernández-Caballero, and José Miguel Latorre. 2019. Emotion Detection in Aging Adults Through Continuous Monitoring of Electro-Dermal Activity and HeartRate Variability. In Int. Work-Conference on the Interplay Between Natural and Artificial Computation. Springer, 252-261.

[17] Arindam Ghosh, Morena Danieli, and Giuseppe Riccardi. 2015. Annotation and prediction of stress and workload from physiological and inertial signals. In 2015 37th Annual International Conference of the IEEE Engineering in Medicine and Biology Society (EMBC). IEEE, 1621-1624.

[18] G. Giannakakis, D. Grigoriadis, K. Giannakaki, O. Simantiraki, A. Roniotis, and M. Tsiknakis. 2019. Review on psychological stress detection using biosignals. IEEE Transactions on Affective Computing (2019).

[19] Alireza Golgouneh and Bahram Tarvirdizadeh. 2019. Fabrication of a portable device for stress monitoring using wearable sensors and soft computing algorithms. Neural Computing and Applications (2019), 1-23.

[20] Rosenberger M Grandner M. 2019. Actigraphic sleep tracking and wearables: Historical context, scientific applications and guidelines, limitations, and considerations for commercial sleep devices. In Sleep and Health. Elsevier, 147-157.

[21] Juan Lorenzo Hagad, Kenichi Fukui, and Masayuki Numao. 2019. Deep visual models for EEG of mindfulness meditation in a workplace setting. In International Workshop on Health Intelligence. Springer, 129-137.

[22] BP Harne, Y Bobade, RS Dhekekar, and A Hiwale. 2019. SVM classification of EEG signal to analyze the effect of OM Mantra meditation on the brain. In 2019 IEEE 16th India Council International Conference (INDICON). IEEE, 1-4.

[23] Cheng He, Yun-jin Yao, and Xue-song Ye. 2017. An emotion recognition system based on physiological signals obtained by wearable sensors. In Wearable sensors and robots. Springer, $15-25$.

[24] RT Hurlburt and CL Heavy. 2002. Interobserver reliability of descriptive experience sampling. Cognitive Therapy and Research 26, 1 (2002), 135-142.

[25] Eiman Kanjo, Eman MG Younis, and Chee Siang Ang. 2019. Deep learning analysis of mobile physiological, environmental and location sensor data for emotion detection. Information Fusion 49 (2019), 46-56.

[26] A Léonard, S Clément, C-D Kuo, and MU Manto. 2019. Changes in Heart Rate Variability During Heartfulness Meditation: A Power Spectral Analysis Including the Residual Spectrum. Frontiers in cardiovascular medicine 6 (2019), 62.

[27] M Maier, C Marouane, and D Elsner. 2019. DeepFlow: Detecting Optimal User Experience From Physiological Data Using Deep Neural Networks. In Proc. of the 18th Int. Conf. on Autonom. Agents and MultiAgent Sys. Int. Found. for Autonom. Agents and Multiagent Sys., 2108-2110.

[28] F Mendonca, SS Mostafa, AG Ravelo-García, F Morgado-Dias, and T Penzel. 2018. A review of obstructive sleep apnea detection approaches. IEEE journal of biomedical and health informatics 23, 2 (2018), 825-837.

[29] Andre Matthias Müller, Nan Xin Wang, Jiali Yao, Chuen Seng Tan, Ivan Cherh Chiet Low, Nicole Lim, Jeremy Tan, Agnes Tan, and Falk MüllerRiemenschneider. 2019. Heart rate measures from wrist-worn activity trackers in a laboratory and free-living setting: validation study. FMIR mHealth and uHealth 7, 10 (2019), e14120.

[30] H Murakami, R Kawakami, S Nakae, Y Yamada, Y Nakata, and et al. 2019. Accuracy of 12 Wearable Devices for Estimating Physical Activity Energy Expenditure Using a Metabolic Chamber and the Doubly Labeled Water Method: Validation Study. In $7 M I R$ mHealth and uHealth.

[31] B. Nakisa, M. N. Rastgoo, A. Rakotonirainy, F. Maire, and V. Chandran. 2018. Long short term memory hyperparameter optimization for a neural network based emotion recognition framework. IEEE Access 6 (2018), 49325-49338.

[32] Grzegorz J Nalepa, Krzysztof Kutt, Barbara Giżycka, Paweł Jemioło, and Szymon Bobek. 2019. Analysis and Use of the Emotional Context with Wearable Devices for Games and Intelligent Assistants. Sensors 19, 11 (2019), 2509.

[33] Sami Nikkonen, Isaac O Afara, Timo Leppänen, and Juha Töyräs. 2019. Artificial neural network analysis of the oxygen saturation signal enables accurate diagnostics of sleep apnea. Scientific reports 9, 1 (2019), 1-9.

[34] Selena R. Pasadyn, Mohamad Soudan, Marc Gillinov, Penny Houghtaling, Dermot Phelan, Nicole Gillinov, Barbara Bittel, and Milind Y. Desai. 2019. Accuracy of commercially available heart rate monitors in athletes: a prospective study. Cardiovascular Diagnosis and Therapy 9, 4 (2019).

[35] Reddipogu Pavani and Akshay Berad. 2019. Study of Effect of Meditation on Galvanic Skin Response in Healthy Individuals. International fournal of Medical and Biomedical Studies 3, 4 (2019).

[36] JM Peake, G Kerr, and JP Sullivan. 2018. A critical review of consumer wearables, mobile applications, and equipment for providing biofeedback, monitoring stress, and sleep in physically active populations. Frontiers in physiology 9 (2018), 743.

[37] S Pham, D Yeap, and et al. 2020. Wearable Sensor System to Monitor Physical Activity and the Physiological Effects of Heat Exposure. Sensors 20, 3 (2020).

[38] S. Saganowski, A. Dutkowiak, A. Dziadek, M. Dziezyc, J. Komoszynska, W. Michalska, A. G. Polak, M. Ujma, and P. Kazienko. 2020. Emotion Recognition Using Wearables: A Systematic Literature Review - Work-in-progress. In 2020 Int. Conf. on Perv. Comp. and Comm. Workshops. IEEE, 1-6.

[39] P. Schmidt, A. Reiss, R. Duerichen, C. Marberger, and K. Van Laerhoven. 2018. Introducing WESAD, a Multimodal Dataset for Wearable Stress and Affect Detection. In Proc. of the 2018 on Int. Conf. on Multimodal Interaction. ACM, 400-408.

[40] P. Schmidt, A. Reiss, R. Dürichen, and K. Van Laerhoven. 2018. Labelling Affective States in the Wild: Practical Guidelines and Lessons Learned. In The 2018 ACM Int. Joint Conf. and 2018 Int. Symp. on Pervasive and Ubiquitous Comp. and Wearable Comp. ACM, 654-659.

[41] F Setiawan, SA Khowaja, AG Prabono, and et al. 2018. A Framework for Real Time Emotion Recognition Based on Human ANS Using Pervasive Device. In 2018 IEEE 42nd Annual Comp. Software and App. Conf., Vol. 1. IEEE, 805-806.

[42] H. Sharma, R. Raj, and M. Juneja. 2019. EEG signal based classification before and after combined Yoga and Sudarshan Kriya. Neurosc. letters 707 (2019), 134300.

[43] Saul Shiffman, Arthur A Stone, and Michael R Hufford. 2008. Ecological Momentary Assessment. Annu. Rev. Clin. Psychol. 4 (2008), 1-32.

[44] M Shoaib, S Bosch, OD Incel, and et al. 2014. Fusion of Smartphone Motion Sensors for Physical Activity Recognition. Sensors 14, 6 (2014), 10146-10176.

[45] MZ Soroush, K Maghooli, SK Setarehdan, and AM Nasrabadi. 2017. A Review on EEG signals based emotion recognition. Int. Clinic. Neurosc. Four. 4, 4 (2017), 118.

[46] J Wei and J Boger. 2019. You Are How You Sleep: Personalized Sleep Monitoring Based on Wrist Temperature and Accelerometer Data. In 13th Int. Conf. on Perv. Comp. Tech. for Healthcare-Demos and Posters. European Alliance for Innovation.

[47] Bin Yu, Mathias Funk, Jun Hu, and Loe Feijs. 2018. Unwind: a musical biofeedback for relaxation assistance. Behaviour \& Inform. Technology 37, 8 (2018), 800-814.

[48] Roberto Zangróniz, Arturo Martínez-Rodrigo, María T López, José Manuel Pastor, and Antonio Fernández-Caballero. 2018. Estimation of mental distress from photoplethysmography. Applied Sciences 8, 1 (2018), 69.

[49] R Zangróniz, A Martínez-Rodrigo, J Pastor, and et al. 2017. Electrodermal activity sensor for classification of calm/distress condition. Sensors 17, 10 (2017), 2324 